\title{
Formación inicial de maestros en promoción de la lectura y la literatura en España desde la perspectiva del profesorado universitario
}

Carmen Álvarez-Álvarez y Julián Pascual-Díez

\section{RESUMEN}

Este artículo analiza la situación actual de la formación inicial de los futuros docentes para desarrollar la promoción lectora y literaria en la escuela primaria. Han participado 73 profesores responsables de la didáctica de la lectura y la literatura de diversas universidades españolas. Se constatan carencias en la formación lectora-literaria en los planes de estudios de magisterio, así como condicionantes positivos y negativos de la formación lectora del alumnado universitario. Se concluye con propuestas de trabajo para hacer que la didáctica de la lectura y la literatura en el aula universitaria sea más formativa, atractiva e intensa.

Palabras clave: desarrollo del profesor, magisterio, lectura, España.

Carmen Álvarez-Álvarez

Española. Doctora en Pedagogía, Universidad de Oviedo, España. Profesora contratada doctora interina en la Universidad de Cantabria, España. Temas de investigación: animación a la lectura, clubes de lectura, organización escolar, innovación educativa. 
Formação inicial de professores no fomento da leitura e da literatura na Espanha dentro da perspectiva do professorado universitário

\section{RESUMO}

Este artigo analisa a situação atual da formação inicial dos futuros docentes para desenvolver o incentivo à leitura e à literatura na escola. Participaram 73 professores responsáveis da didática de leitura e literatura de diversas universidades espanholas. Constatou-se carências na formação leitora-literária nos planos de estudos de magistério, assim como condicionantes positivas e negativas da formação leitora dos alunos universitários. Conclui-se com propostas de trabalho para fazer que a didática da leitura e da literatura no ambiente universitário seja mais formativa, atrativa e intensa.

Palavras chave: desenvolvimento do professor, magistério, leitura, Espanha.

Initial teacher training in the promotion of reading and Literature in Spain from the perspective of university teachers

\section{ABSTRACT}

This article analyzes the current situation of the initial training of future teachers to develop the promotion of reading and literature in primary school. Seventy-three teachers took part in this research, all of them responsible for the didactics of reading and literature in various Spanish universities. The academic staff showed there are shortcomings in reading and literature education in teacher training curricula, as well as positive and negative conditioning factors in the reading education of university students. The study concludes with work proposals to make the didactics of reading and literature in the university classroom more formative, attractive and intense.

Key words: teacher development, teaching, reading, Spain. 
$\mathrm{U}$ no de los objetivos que tiene que afrontar el profesorado con los escolares es el desarrollo de la promoción lectora y literaria. Éste es un reto especialmente arduo para el profesorado recién titulado, que carece de práctica en el trato con niños y de experiencia en materia de animación a la lectura. En este sentido, contar con una buena formación inicial en la materia se hace fundamental para saber cómo actuar con profesionalidad, tal como ha demostrado la investigación (Caldera, Escalante y Terán, 2010; Hoeft, 2012; Leland, 2013; Munita, 2013; Granado y Puig, 2014).

Sin embargo, por nuestra experiencia, hemos constatado que esta formación inicial presenta notables carencias. Por ello, hemos decidido realizar un estudio empírico consultando a profesorado universitario que imparte materias relacionadas con la didáctica de la lengua y la literatura (DLL) en diferentes planes de estudio de Magisterio de toda la geografía española. El objetivo es conocer en qué estado se encuentra la formación inicial de maestros en este ámbito y plantear propuestas de mejora.

\section{Referentes teóricos}

Es de sobra conocido el debate sobre el papel que puede desempeñar la escuela en el fomento de la lectura y las dificultades que debe abordar para promover el placer por la lectura literaria y el desarrollo del hábito lector. Resulta imprescindible que los dos grandes objetivos lectores que tiene que afrontar la escuela: la lectura instrumental y obligatoria (leer para aprender) y la lectura como placer y entretenimiento no se planteen de forma tal que la primera acabe por anular el crecimiento y desarrollo de la segunda o que ésta quede marginada al tiempo extraescolar, especialmente si se tiene en cuenta que la lectura recreativa regular resulta beneficiosa tanto para el desarrollo de la competencia lingüística como para el aprendizaje de otras materias (Merga, 2107).

La animación a la lectura debe contribuir a la comprensión lectora, cuyo dominio debería ser a su vez el mejor modo de animar a leer, pues lo que está en juego en ambos casos es alentar el progreso y la satisfacción del lector (Mata, 2008). Por consiguiente, se hace necesario un planteamiento global en el marco de una didáctica integradora de la lectura. Se trata de abordar de forma equilibrada el saber con el querer leer evitando una confrontación entre ambos objetivos. Y es que tal y como apunta Colomer: "promover la lectura y enseñar a leer son los dos ejes sobre los que discurre la innovación en la enseñanza de la literatura" (Colomer, 2001: 17).

El profesor de literatura es el enseñante, pero también es, en alguna medida, un mediador en lectura, aunque no el único, al menos en las primeras edades (Cerrillo, 2007). Sin embargo, la labor de mediación, además de saberes y habilidades para promover el desarrollo de la competencia lectora y literaria en los escolares, exige un bagaje lector personal que es claramente insuficiente en muchos de los futuros maestros: la investigación está poniendo de relieve que en las universidades: 1) hay un creciente número de estudiantes "aletrados", es decir, que eligen no leer por carecer de motivación intrínseca 2) que los hábitos lectores de los estudiantes universitarios son insuficientes y 3) son frecuentes las quejas del profesorado sobre las deficiencias en lectura del alumnado de reciente ingreso a la universidad, a pesar de que es ésta una competencia clave para acceder a saberes y desarrollar capacidades propias de las enseñanzas universitarias (Díaz, Bar y Ortiz, 2015; Felipe, 2016; Vidal-Moscoso y Manríquez-López, 2016). Esta situación generalizada, parece reproducirse en los estudios de Magisterio en España.

En efecto, tal y como afirman Colomer y Munita (2013: 38), aunque el planteamiento generalizado de muchos programas de formación del profesorado parten de la idea de que los futuros maestros son lectores expertos, la realidad evidencia lo contrario, "tanto a nivel de sus hábitos personales de lectura" como en lo que se refiere a "sus saberes y su capacidad de interpretación sobre los textos literarios". 
Trabajos como los de Larrañaga, Yubero y Cerrillo (2008); Cremin, Mottram, Bearne y Goodwin (2008); Díaz Armas (2008); Granado (2014); Tabernero (2013); Munita, (2014) y Applegate et al. (2014) constatan e ilustran sobradamente esta realidad. Por ejemplo, el trabajo de Larrañaga, Yubero y Cerrillo (2008), centrado en estudiantes universitarios y el de Granado (2014), sobre estudiantes de Magisterio, ponen de manifiesto el alto porcentaje de ellos que se declaran no lectores o que presentan muchas carencias en sus hábitos de lectura.

Munita (2014), por su parte, subraya cómo, además, muchos de ellos consideran que navegan por corpus literarios amplios que en realidad se basan en lecturas discontinuas mayoritariamente de best-sellers, lo que origina una competencia literaria ilusoria. Estos datos resultan preocupantes y acaso les imposibilita para ejercer la tarea de mediadores. Applegate et al. (2014) encontraron niveles sorprendentemente bajos de entusiasmo por la lectura en los futuros docentes, llamados a inspirar a sus alumnos un amor por ésta que ellos mismos no poseen.

En este contexto es imprescindible replantear en profundidad las líneas de actuación didáctica que teóricamente cabría esperar, orientadas principalmente a analizar cómo favorecer en el aula procesos de enseñanza-aprendizaje que promuevan el desarrollo de la competencia lectora y literaria y un compromiso e implicación afectiva de los escolares hacia la literatura. La educación literaria incluye el saber, el saber hacer, el saber cómo se hace, el opinar y el sentir (Ballester e Ibarra, 2009). Además de este objetivo que, obviamente, sigue siendo irrenunciable, "la experiencia lectora de los futuros maestros, así como sus capacidades para dinamizar los textos literarios en el aula, se yergue como uno de los nuevos desafíos que debe enfrentar la didáctica de la literatura" (Colomer y Munita, 2013: 38). En términos parecidos se expresa Tabernero (2013: 48) cuando subraya que resulta necesario propiciar "el encuentro del lector con el texto desde los presupuestos de la discusión literaria que permitan la «recreación» del lector [...] con el ánimo de formar esos lectores reflexivos". En definitiva, entre los objetivos principales que los docentes de DLL deben plantearse en sus clases estará el de modificar la idea de que la literatura es sólo para estudiarla:

No tiene nada de sorprendente que tan pocos, aun de los graduados universitarios, se hayan formado el hábito de volcarse a la literatura por placer y discernimiento. La novela, la obra de teatro, el poema, han sido convertidos para ellos en algo sobre lo que tienen que saber, algo que hay que resumir, analizar o definir (Rosenblatt, 2002: 84).

Otro problema importante asociado a la formación inicial del profesorado es el relacionado con las concepciones e ideas previas de los estudiantes de Magisterio sobre lo que es leer, la percepción que tienen sobre la literatura, y especialmente la imagen prefijada que vincula la lectura literaria a una tarea escolar o asignatura. A esta circunstancia se unen habitualmente ciertas ideas preconcebidas en torno a la literatura infantil y juvenil (LIJ) que distorsionan el sentido de ésta: prejuicios ideológicos, vinculación de la calidad de los textos a la enseñanza en valores, temáticas e ilustraciones que se consideran o no adecuadas, criterios de selección del corpus... (Díaz Armas, 2008; Tabernero, 2013), potencialidades de sus alumnos en las primeras edades (Gutiérrez-Fresneda, Díez-Mediavilla y Jiménez-Pérez, 2017). Ello obliga al profesorado de DLL, en palabras de Tabernero (2013), a tener que deconstruir para construir.

Pero además de considerar los hábitos lectores y la actitud hacia la literatura por parte de los futuros maestros, el profesorado de DLL tiene que realizar una autocrítica seria y rigurosa sobre su propia formación y sobre sus concepciones y creencias didácticas. Sería insuficiente, además de injusto, hacer recaer en las carencias que presentan muchos de los futuros docentes la responsabilidad del mayor o 
menor éxito de las prácticas de educación lectora que puedan desarrollarse en el futuro, sin contemplar otros factores.

Diversos trabajos constatan, tal y como afirman Granado y Puig (2014: 93), que la relación personal del profesorado con la lectura por un lado "influye en el tipo de prácticas de educación lectora que ofrecen en su aula y, por otro, en las creencias que sobre el valor de la lectura mantienen y transmiten a sus estudiantes". Esta influencia puede producirse, aunque sea con diferente intensidad, en todas las etapas educativas. Por ello sería conveniente que los docentes que imparten DLL en la universidad analizaran qué tipo de relación mantienen con la lectura y qué creencias explícitas o implícitas transmiten a sus estudiantes, futuros docentes, a través de sus palabras y de su propia acción docente.

La dimensión práctica de la formación es otra de las cuestiones clave. En la formación literaria del profesorado, la combinación de teoría y práctica es una necesidad porque posibilita que los conocimientos adquiridos se sepan aplicar y justificar ante supuestos de situaciones reales (Cerrillo, 2007). Y es que tal y como subraya Mendoza, la necesidad de investigar en DLL debe realizarse "en, desde y para el aula" (Mendoza, 2011: 44). Esta realidad debería inspirar el enfoque formativo de las clases en la universidad con el fin de propiciar tareas en las que los futuros docentes tengan que interrelacionar conocimientos teóricos y conocimientos prácticos.

Durante los últimos años el proceso lector y las modalidades textuales han cambiado y han hecho más compleja la manera de leer con la aparición de los textos multimodales en el espacio 2.0, que hacen precisa una alfabetización múltiple y que plantean nuevos retos en la formación del lector (Arbonés et al., 2015). Como explica Serafini (2012), los textos del siglo XXI exigirán un profesorado capaz de adoptar nuevas habilidades, estrategias y planteamientos pedagógicos para ayudar a los estudiantes, ya que los textos multimodales requieren lectores que naveguen, diseñen, interpreten y analicen textos de una forma más compleja e interactiva.

Este análisis quedaría incompleto si no consideráramos la influencia de los aspectos relacionados con el contexto actual de los planes de estudio y las condiciones de trabajo del profesorado. Respecto a los nuevos planes de estudio, cabe destacar la necesidad de una adecuada profesionalización docente que viene exigida por el Espacio Europeo de Educación Superior (EEES). Tal y como subraya Camps (2012: 38):

En España, la adaptación de los planes de estudios de formación del profesorado a las directrices emanadas de la Unión Europea, el denominado "Plan Bolonia", se realizó sin debates profundos sobre los perfiles profesionales de los docentes ante las demandas actuales, por lo menos en lo que respecta a la formación lingüística de la ciudadanía y de los docentes.

Además, el análisis de la realidad evidencia la escasa presencia de materias y de créditos asignados a la DLL, de la literatura en general y, más específicamente, de la LIJ en la formación de maestro (Ballester, 2015). Parece, por tanto, muy relevante que los planes de estudios de Magisterio o que las facultades a través de otras estrategias se esfuercen al máximo en formar a los futuros profesores adecuadamente en materia de lectura y literatura (Leland, 2013).

Por otro lado, no se pueden obviar las circunstancias actuales que vive buena parte del profesorado universitario como consecuencia de las medidas adoptadas con la crisis económica reciente: incremento de la carga horaria, precariedad, aumento de figuras laborales temporales, problemas asociados a la carrera docente y acreditaciones, etcétera ( , 2017). Estos condicionantes inciden enormemente en la formación y en la cultura profesional de los docentes responsables de la formación de maestros.

Así como se ha investigado bastante sobre el alumnado y con el alumnado, por el contrario, no hay muchos estudios en nuestro país donde el foco 
sea el profesorado universitario responsable de la formación impartida en los estudios de Magisterio. En esta situación tan poco propicia que muestran las investigaciones, pensamos que el profesorado tiene mucho que decir sobre la situación actual en la formación didáctica de la lectura y la literatura y sobre cómo se puede mejorar.

\section{Objetivos}

Nuestro objetivo general es saber en qué estado se encuentra la formación inicial de maestros en lectura en España en estos momentos y plantear propuestas de mejora. Nuestros objetivos específicos guardan relación con conocer:

- La presencia de la formación lectora-literaria en los actuales planes de estudio de Magisterio en España.

- Los condicionantes positivos y negativos de la formación lectora del alumnado en sus etapas de escolarización y en la actualidad.

- Las fortalezas y carencias que tienen para promover la lectura en la escuela.

- Propuestas de mejora para el fortalecimiento de la formación inicial lectora-literaria de los futuros maestros.

\section{Material y Métodos}

Para abordar estos objetivos, hemos considerado que lo más adecuado era plantear un cuestionario abierto a informantes-clave, docentes universitarios responsables de impartir materias relacionadas con la formación didáctica en lectura y literatura. Deseábamos que este cuestionario fuese breve pero que nos pudiese ofrecer información generosa respecto a los temas objeto de investigación. Así, decidimos hacerlo abierto, sin limitar la extensión de cada ítem (5, al final). Se envió una primera versión del cuestionario (cuestionario piloto) a un reducido número de profesores especialistas con reconocido prestigio en el área de conocimiento. Tras este envío, procedimos a hacer ligeras modificaciones en uno de los ítems. El cuestionario definitivo se envió personalmente por correo electrónico a todo el profesorado que imparte docencia en materias afines a la didáctica de la lectura y la literatura en los estudios de Magisterio en Educación Primaria de todas las universidades españolas. La denominación de las asignaturas no es homogénea entre las distintas universidades. A la más habitual de "Didáctica de la lengua y la literatura", le siguen otras como: "Didáctica de la lengua oral y escrita", "Educación literaria", "Literatura infantil y juvenil", "Formación literaria", "Enseñanza y aprendizaje de competencias comunicativas", "Literatura, lectura y creatividad", "Biblioteca y animación lectora". Para poder determinar la población objeto de estudio, fue preciso analizar todas las guías docentes del curso 2016-2017 y comprobar así si la materia impartida se ajustaba al propósito de este estudio.

La tasa de respuestas al cuestionario ha sido satisfactoria ya que, dado su carácter cualitativo, no se perseguía tanto que fuera estadísticamente significativa, como que fuera representativa y proporcionada de las diferentes universidades y perfiles profesionales docentes. El número de sujetos de la muestra participante supera el 30\% del total de docentes que componen la población. Se han obtenido 73 respuestas de docentes pertenecientes a 45 universidades distintas, ubicadas en las 17 comunidades autónomas de España, con mayor representación en aquellas donde hay más universidades; de instituciones públicas y privadas, de hombres y mujeres, y de docentes más y menos experimentados. Los docentes que han participado en este estudio se distribuyen del modo que se indica en la tabla 1.

El cuestionario empleado se compone de las siguientes preguntas abiertas:

- Dentro de los estudios de grado de Magisterio de tu universidad, ¿cómo valoras la importancia que se le otorga a la lectura, a la literatura y a sus didácticas en los planes de estudios actuales? 
- Qué aspectos positivos y negativos en la educación primaria y secundaria recibida por el alumnado de Magisterio condicionan tu trabajo en los aspectos relacionados con la didáctica de la lectura y la literatura.

- ¿Consideras que el alumnado de Magisterio termina sus estudios con una buena formación para promover la lectura en la escuela cuando sean maestros/as? Cuáles son las principales carencias o deficiencias que observas en este terreno.
- Qué se podría mejorar en la formación de los estudiantes de Magisterio para que pudieran afrontar más satisfactoriamente los procesos de enseñanza-aprendizaje de la lectura y el desarrollo de la competencia lectora y literaria en la escuela primaria.

- Escribe - si lo deseas - otras reflexiones o propuestas relacionadas con la formación inicial de los maestros en didáctica de la lectura y literatura que ayuden a mejorar nuestro trabajo sobre estos ámbitos desde la universidad.

Tabla 1. Número de colaboradores por comunidad autónoma y número de universidades implicadas

\begin{tabular}{|c|c|c|}
\hline Comunidad autónoma & $\begin{array}{l}\text { Número de } \\
\text { participantes }\end{array}$ & $\begin{array}{c}\text { Número de universidades } \\
\text { implicadas }\end{array}$ \\
\hline Galicia & 6 & 4 \\
\hline Asturias & 1 & 1 \\
\hline Cantabria & 1 & 1 \\
\hline País Vasco & 3 & 1 \\
\hline Castilla León & 6 & 4 \\
\hline Madrid & 6 & 6 \\
\hline Navarra & 1 & 1 \\
\hline La Rioja & 2 & 1 \\
\hline Aragón & 5 & 1 \\
\hline Cataluña & 9 & 6 \\
\hline Comunidad valenciana & 10 & 6 \\
\hline Murcia & 3 & 2 \\
\hline Andalucía & 10 & 6 \\
\hline Castilla La Mancha & 4 & 1 \\
\hline Extremadura & 1 & 1 \\
\hline Islas Baleares & 1 & 1 \\
\hline Islas Canarias & 4 & 2 \\
\hline Total & 73 & 45 \\
\hline
\end{tabular}


Tras la recogida de información, se organizaron y analizaron los datos, siguiendo pautas propias del análisis de contenido, ya que todos ellos son de tipo cualitativo. En primer lugar, se estableció una primera categorización de las respuestas dadas a cada pregunta, la cual fue evolucionando a medida que se abordaba la lectura sistemática de los cuestionarios. Posteriormente, se realizó un vaciado minucioso de éstos, siguiendo un procedimiento de triangulación, en el que tras la lectura de cada cuestión, los autores de este estudio consensuaban la decisión de incluir las respuestas en una de las categorías previamente fijadas o de crear una nueva. Finalmente, se procedió a una reducción de las categorías establecidas para poder organizarlas y evitar la dispersión de datos.

\section{Resultados}

Los resultados se han organizado en torno a cuatro grandes categorías, que coinciden con los objetivos específicos señalados anteriormente. Cuando se incluyan citas textuales de los testimonios de los docentes, se empleará un código que indica el sexo y un número identificativo (ejemplo: profesor 17; profesora 25).

\section{Presencia de la formación lectora-literaria en el Plan de Estudios de Magisterio}

Una vez analizadas las 73 respuestas ofrecidas por el profesorado de las diferentes universidades españolas, se constata un acuerdo generalizado acerca de la escasa importancia otorgada a la lectura en los estudios de Magisterio. Para 53 docentes, la formación en didáctica de la lectura y la literatura es claramente insuficiente, mientras que para 20 es aceptable, aunque podría mejorarse. Nadie ha considerado que la situación sea buena. Testimonios como el siguiente se repiten:

El plan de estudios del grado, tanto de Primaria como de Infantil, reduce notablemente el número de créditos dedicados a la literatura, a la lengua castellana y a la didáctica de la lengua. Si están interesados, no duden en pedirme las cifras que prueban esta pérdida. Por lo tanto considero que en nuestro plan de estudios se le da una importancia insuficiente. Los mismos alumnos me dicen muchas veces: esta asignatura tenía que ser anual (Profesora 33).

También hay quienes señalan diferencias en el planteamiento de la formación lecto-literaria entre los Planes de Estudio de Educación Infantil o Educación Primaria, incluso dentro de la misma universidad.

En mi universidad no hay una asignatura específica de Lengua en el Grado de Infantil, y en el de Primaria no aparece hasta el curso $3^{\circ}$. Respecto a la lectura, en $4^{\circ}$ de Infantil está la asignatura "Lectura, escritura y Literatura infantil", y en $4^{\circ}$ de Primaria "Literatura y educación" (Profesor 29).

El profesorado valora muy negativamente las horas de docencia asignadas a algunas materias implicadas en la capacitación didáctica, así como el hecho de que muchas de ellas sean optativas, porque consideran que todo el alumnado debería cursarlas obligatoriamente: "Son asignaturas con una carga lectiva anecdótica y en algunos casos optativas o de libre elección, por lo que los futuros mediadores pueden completar sus estudios sin conocer prácticamente nada de la evolución de la literatura" (Profesora 1).

También varios profesores califican muy negativamente las condiciones estructurales a las que están sometidas las universidades: dotación de profesorado estable, horarios insuficientes e irregulares, elevado número de estudiantes por aula, etcétera.

Mi experiencia ha sido que el tiempo ha sido escaso para cubrir todo el temario y trabajar la oralidad, la escritura y el cuento. Más escaso se queda si se pretende poner en práctica la teoría impartida debido al número tan elevado de alumnos en las aulas (Profesora 24). 
Otro aspecto relevante que señalan varios profesores son los programas de estas materias. Para algunos, la presencia de asignaturas de DLL no asegura que la formación que se imparta sea la adecuada, tratándose de la capacitación de maestros: "Los programas de estas materias parecen estar diseñados para filólogos y no para maestros" (Profesora 2).

Otras personas reclaman mayor implicación de los decanatos en la oferta de actividades complementarias en didáctica de la lectura y la literatura: "Es claramente insuficiente, por el número de créditos que se le dedican, así como por la falta de actividades complementarias a la docencia reglada que se promueven en el Decanato" (Profesora 5).

En este sentido, hay universidades más activas en las que el área de DLL ya se está planteando o se están elaborando planes de especialización (menciones, másteres, etcétera) en Narrativa, Biblioteca Escolar, LIJ, etcétera: "Destaco la existencia en uno de nuestros campus de una Mención para los maestros de Primaria (Lengua, lectura y multiculturalidad) que intenta suplir estas carencias" (Profesor 26).

A la vista de los testimonios recogidos, resulta preciso introducir muchas mejoras en el diseño de los planes de estudio en las materias relacionadas con la didáctica de la lectura y de la literatura.

\section{Condicionantes positivos y negativos de la formación lectora del alumnado}

El segundo foco del estudio guarda relación con los condicionantes positivos y negativos de la formación lectora del alumnado en sus etapas de escolarización previa y en la actualidad. Si bien es cierto que es posible identificar aspectos positivos y negativos en las respuestas, predominan con mayor abundancia los negativos y muy especialmente el hecho de que el alumnado no sea lector habitual. A ello se refieren prácticamente la totalidad de los participantes en el estudio. En síntesis, el profesorado ha señalado los siguientes aspectos positivos y negativos:

\section{Aspectos positivos}

- En todas las aulas hay un reducido número de alumnos que muestra interés hacia las cuestiones relacionadas con la lectura literaria en la infancia.

- Hay alumnado que está muy motivado, se esfuerza y trabaja mostrando preocupación y motivación por aprender, participación activa, etcétera.

- Una parte de los estudiantes manifiestan interés por informarse y descubrir obras o propuestas para el trabajo en el aula de Infantil y Primaria. Para algunos alumnos es ilusionante descubrir "ese mundo que se han perdido".

- Hay temas que les interesan como la lectura de algunos textos literarios, la creación artística mediante la reescritura creativa de textos, la narración oral de cuentos y la combinación de literatura y tecnologías de la información y la comunicación (TIC).

- En ocasiones, el alumnado sorprende con propuestas creativas basadas en la superación de las actividades que vivieron en su infancia o adolescencia y que no habían resultado exitosas.

- Algunos alumnos tienen buen recuerdo de algunas obras leídas en Educación Primaria.

- Entienden y defienden la importancia de la motivación para leer. Conocen bien el ideal educativo, aunque muchos no lo cumplan.

- Tienen agilidad mental, fruto de que están habituados a estar atentos a varias cosas a la vez.

- A través de plataformas como Blackboard realizan trabajos on-line de buena calidad. Suele haber cooperación entre el alumnado.

- Algunos han tenido buenos modelos lectores en sus vidas: familiares o docentes. En los últimos años parece que el alumnado inicia magisterio con mayor conocimiento de la literatura contemporánea.

- Hay alumnado que ha tenido experiencias de educación lecto-literaria interesantes en 
Educación Primaria y Secundaria (talleres, recitales, proyectos, actividades de animación lectora...).

- Después de pasar por las asignaturas de DLL, muchos van modificando su percepción sobre la importancia de la lectura y la formación literaria.

\section{Aspectos negativos}

- Escaso hábito de lectura del alumnado (de textos literarios y de otro tipo).

- Escasa competencia lectora, literaria y lingüística. El nivel es más bajo de lo que sería esperable en un futuro maestro.

- La asociación entre lectura y literatura con una actividad obligatoria y aburrida. Animadversión hacia la asignatura de Lengua Castellana y Literatura (desapego por la propia cultura y su idioma) y, por tanto, hacia su didáctica.

- Les han obligado a leer libros que no les han gustado. No les han dado la oportunidad de elegir sus lecturas. No les han dirigido a la hora de conocer diferentes temáticas con las que podrían identificarse.

- Importantes carencias en la educación literaria recibida; nunca han estudiado LIJ con anterioridad. No tienen adquiridas ciertas nociones de literatura básicas: autores, tendencias, obras, etcétera.

- Poseen un enfoque excesivamente histórico, memorístico y tradicional de la literatura. Ven la materia como una nómina de autores y títulos y no como algo vivo, dinámico y emocionante.

- Carencia de referentes literarios y conceptos básicos en la materia. Desconocimiento de los clásicos. Concepción utilitarista de la LIJ.

- Dificultad para seleccionar obras literarias con calidad: poca sensibilidad estética para elegir con criterio. Ausencia de una buena capacidad crítica.

- Creencia en ciertos casos de que sus conocimientos tienen que ser los mismos que los de un niño de Educación Primaria.

- Problemas de expresión y comprensión escrita. Escaso hábito de escritura creativa y académica. Falta de léxico. Fallos básicos de redacción y ortografía.

- Bajo nivel de profundidad de sus razonamientos. Rozan aspectos superficiales de lo que leen y escriben. Carencias en comprensión lectora.

- Pertenecen a una generación digital: están acostumbrados a leer y escribir de forma discontinua en pantalla y les cuesta leer un texto continuo.

Aunque a la luz de las afirmaciones anteriores, existen algunos condicionantes positivos en la tarea docente sobre los que podrían cimentarse planteamientos didácticos consistentes, los aspectos negativos pesan mucho y constituyen un escollo difícil de afrontar en el contexto actual.

\section{Fortalezas y debilidades para promover la lectura en la escuela}

El tercer aspecto analizado aborda las fortalezas y carencias que tiene el alumnado de Magisterio para poder promover la lectura en la escuela en un futuro. El profesorado universitario se muestra muy pesimista, las fortalezas tienen que ver con el provecho que hayan sacado en las escasas asignaturas que cursan vinculadas a este campo de estudio. Sin embargo, las carencias superan con creces a las fortalezas. Únicamente cuatro de los 71 profesores colaboradores consideran que el alumnado sale de la carrera con una buena formación para afrontar el reto de formar a la infancia adecuadamente; 21 consideran que la formación con la que salen es aceptable y 48 afirman con rotundidad que las carencias son muchas. Se destaca nuevamente la falta de hábito lector del alumnado como la principal barrera, algo que a su juicio no se puede superar cursando simplemente unas asignaturas de un plan de estudios. En 
concreto, las fortalezas y debilidades que se destacan son las siguientes:

\section{Fortalezas}

- Trabajo duro en las asignaturas durante los cursos de muchos profesores que tratan de suplir carencias de todo tipo. El alumnado toma conciencia de la importancia de la lectura como elemento formativo de los niños.

- Trabajo coordinado del profesorado de algunas áreas de DLL para superar obstáculos comunes y favorecer un mejor aprovechamiento del tiempo de las materias.

- La formación de maestros es una tarea continua y permanente y en las materias de DLL se tratan de sentar las bases para que los futuros docentes prosigan después con su proceso formativo autónomamente. Existe el convencimiento de que no todo depende de la formación inicial.

- En comunidades con dos lenguas oficiales, el alumnado suele tener más formación en DLL como consecuencia de que duplican materias (Didáctica del castellano y de la lengua propia de la comunidad).

- Algunos (pocos) alumnos en los periodos de Practicum han vivido experiencias de animación a la lectura y han desarrollado estrategias aprendidas durante la carrera, vinculando así teoría y práctica.

- Lectura de algunas obras relevantes y, en ocasiones, comentario de las mismas en el aula universitaria.

- El alumnado se motiva cuando planifica clases, elabora proyectos lectores, crea cuentos, poesías, cómics, canciones, dramatizaciones, etcétera.

- Buena disposición al trabajo docente de la mayoría de estudiantes y al desarrollo profesional docente de bastantes de ellos.

\section{Debilidades}

- Dificilmente pueden contagiar el gusto por la lectura futuros maestros que no leen o leen por obligación, que piensan que la lectura no sirve para nada o que simplemente sirve para transmitir valores. Con frecuencia se quejan de tener que leer.

- Escasez de tiempo para desarrollar conocimientos, prácticas y fomentar la pasión por la lectura. Las asignaturas cursadas relacionadas con la DLL en la carrera tienen pocos créditos. No es posible abordar con profundidad estrategias de promoción lectora.

- Las carencias con las que inician los estudios son muy graves y sus conocimientos en literatura cuando salen de la carrera aún son muy reducidos. La disposición e interés hacia la mejora difiere mucho entre estudiantes. Desconocen obras, autores, géneros y subgéneros. No conocen los clásicos. La oferta editorial es inabarcable y no sabrán seleccionar obras de calidad. Falta lectura crítica.

- Falta de implicación o de formación de algunos profesores universitarios; deficiente dotación de personal estable en el área; falta de cooperación entre las áreas de conocimiento para mejorar la formación lectora; escasez de programas y estrategias adecuadas para potenciar buenos profesionales en el fomento de la lectura y escaso convencimiento por parte de las instituciones universitarias para apostar por la educación lingüística y literaria.

- Tienen dificultad para salirse de modelos instructivos. Los estudiantes están seriamente encorsetados por su bagaje escolar como alumnos. Es más fácil reproducir que crear. Muchos creen que, utilizando los materiales preparados por las editoriales, podrán suplir sus carencias.

- Se necesita mucha más conexión con los centros educativos de Educación Primaria y Secundaria. Hay una enorme distancia entre lo 
que sucede en las escuelas e institutos y el trabajo docente universitario.

Este testimonio recoge en cierta manera el sentir mayoritario del profesorado, aunque con sus ejemplos denota un mayor grado de compromiso con la enseñanza de la materia que el mostrado por algunos participantes.

En lo que a mí respecta, creo que sí proporciono los mimbres para que puedan animar a la lectura, dándoles a conocer propuestas innovadoras, llevándoles al aula propuestas innovadoras realizadas en bibliotecas escolares y bibliotecas infantiles y juveniles [...]. También conocen de primera mano a algunos de nuestros escritores de LIJ más conocidos que nos han visitado y han tenido la oportunidad de trabajar con sus obras y hacer propuestas de animación para el aula de primaria. Las principales deficiencias, lamentablemente, están en que el alumnado llega al aula con un perfil lector muy, muy bajo y eso, como es lógico, hace que, como maestros, si ellos no son lectores, la animación a la lectura es harto difícil (Profesor 3).

\section{Mejoras para el fortalecimiento de la formación lectora-literaria}

Son numerosas las mejoras que el profesorado ha planteado para fortalecer la formación lectora-literaria del alumnado de Magisterio. Las más frecuentes y que se citan con más énfasis son las siguientes:

- Reorganizar los planes de estudio para introducir nuevas asignaturas que refuercen la formación lectora y literaria y ampliar los créditos de las asignaturas del área de conocimiento ya existentes, evitando así que las asignaturas de literatura sean optativas o de libre configuración.

- Realizar una selección del alumnado de Magisterio para que sólo puedan cursar la carrera alumnos con verdadera motivación y vocación y para que los grupos de clase sean más reducidos. Realizar una prueba específica de competencia comunicativa en lengua castellana.

- Conocer e incrementar las relaciones del profesorado de la facultad con organizaciones que promueven la lectura en su entorno: aulas de Educación Infantil y Primaria, bibliotecas, librerías, editoriales, ludotecas, autores de LIJ, clubes de lectura, etcétera. Se precisa una mayor presencia del profesorado universitario en la realidad escolar y más visitas de profesionales que desarrollen prácticas valiosas de lectura en las aulas universitarias.

Algunos docentes, autocríticamente, también se plantean que ellos mismos podrían dar una visión más práctica de sus asignaturas, que en ocasiones falla la coordinación entre profesores o materias, etcétera. Otros, especialmente sensibilizados, han desarrollado iniciativas interesantes, como esta: "He creado un grupo privado de Google para seguir con recomendaciones literarias, dando pautas y consejos para fomentar la lectura, etc. Pienso que esa conexión, aun después de egresados, facilita seguir en contacto con este mundo" (Profesora 16).

Además, el profesorado ha realizado otras propuestas, como las siguientes:

- Aumentar la formación en asuntos específicos: cuenta-cuentos, clases de teatro, estrategias de dinamización lectora, talleres de escritura y de comentario de textos, charlas de especialistas, concursos y premios, ferias del libro, etcétera, para mejorar las habilidades literarias, lingüísticas y comunicativas del alumnado.

- Informar al alumnado de redes de intercambio científicas de difusión a las que puedan vincularse para continuar en formación: páginas web, blogs, asociaciones, fundaciones, revistas especializadas, jornadas, cursos, congresos, etcétera. 
- Desarrollar más experiencias de lectura literaria, para que los estudiantes descubran sus virtudes. Crear clubes de lectura, tertulias literarias, círculos de lectura, etcétera, para adquirir una cultura mayor.

- Llegar a acuerdos para fijar unas obras de literatura universal, clásica y de LIJ, de lectura obligatoria por parte del alumnado a lo largo de la carrera.

- Concientizar a los profesores de Magisterio de todas las áreas de conocimiento sobre la importancia de que un futuro maestro sepa expresarse oralmente y por escrito con corrección, cohesión y coherencia. Podría hacerse un examen final de competencia comunicativa en lengua española previo a la obtención del título.

- Mejorar la dotación y organización de las lecturas de LIJ de las bibliotecas universitarias para que éstas sean accesibles, actuales y relevantes.

- Replantear el enfoque general que se da a la educación literaria en nuestro sistema educativo y trabajar más seriamente la competencia lectora.

- Cuidar la didáctica empleada en las clases universitarias: potenciar la lectura en voz alta de obras, la elaboración de reseñas y comentarios críticos de textos, la lectura de prensa, acudir a la biblioteca, diseñar clases, crear narraciones, interrelacionar lenguajes (literario, pictórico y musical), etcétera, abordando la literatura desde una perspectiva práctica centrada en el trabajo escolar.

La situación parece compleja y los resultados hasta ahora analizados ponen de manifiesto la necesidad de mejoras urgentes. En palabras de uno de los profesores colaboradores:

El lobo no puede guardar las ovejas. El profesorado debe estar formado suficientemente en la lectura y, sobre todo, saber qué estrategias poner en funcionamiento para crear sensibilidad lectora en el niño/a. En definitiva, creerse lo que está haciendo (Profesor 21).

\section{Conclusiones y propuestas de mejora}

A la luz de los resultados, parece confirmarse nuestra hipótesis de partida, según la cual la formación inicial en didáctica de la lectura y literatura del profesorado en formación es débil y hace falta una reforma profunda en varios planos. Quizá lo más destacable sea el tono pesimista que muestran los docentes de DLL, al considerar de forma mayoritaria que los futuros maestros no terminan sus estudios con una formación satisfactoria para desarrollar en el futuro la tarea de promoción lectora. El aspecto que consideran más grave es la falta de hábito lector del alumnado, circunstancia que lastra el trabajo realizado en las asignaturas de DLL. El análisis de estudios en este campo y las valoraciones realizadas por el profesorado universitario que está actualmente en ejercicio nos llevan a plantear siete líneas de actuación que redundarían en mejoras en la formación inicial de los futuros maestros.

1. En primer lugar, se podrían adaptar y adoptar algunas de las propuestas formuladas por el profesorado de DLL participante en el estudio. Especialmente relevantes nos parecen algunas relacionadas con los planes de estudio y las condiciones de trabajo: necesidad de reorganizar los planes de estudio para ampliar los créditos de las asignaturas del área de DLL, realizar una selección del alumnado que accede a los estudios de Magisterio, mejorar la dotación y organización de fondos bibliográficos sobre LIJ en las bibliotecas universitarias, mejorar las condiciones de trabajo del profesorado (mayor estabilidad, reducción del número de alumnos por aula, etcétera), mayor ajuste de los programas de las asignaturas a la finalidad que persiguen, etcétera.

2. Otro aspecto destacable es el de la formación del profesorado responsable de impartir DLL. Algunos docentes participantes en este trabajo llaman la atención 
sobre la falta de formación de ciertos compañeros; en otros casos, echan en falta una mayor implicación y actualización docente para emplear estrategias de promoción lectora más adecuadas en el aula. La formación en LIJ también es otro ámbito donde la preparación del profesorado que imparte DLL resulta insuficiente (Aguilar, 2016). Quizás el impacto de algunos problemas y carencias citados anteriormente ha hecho que el profesorado encuestado no haya destacado el reto didáctico que supone la lectura hipertextual y la lectura literaria en pantallas Así, por ejemplo, Arbonés et al. (2015) ponen el acento en la necesidad de potenciar la formación en los recursos y procedimientos que ofrece la web 2.0 para ampliar la competencia literaria.

3. Es aconsejable que el profesorado de DLL posea un mayor conocimiento sobre la trayectoria lectora de sus alumnos, futuros docentes, con el fin de obtener una información contextual lo más completa posible que le ayude a detectar carencias y necesidades, y poder ajustar así su intervención didáctica. En este sentido, se puede recurrir a entrevistas personales o cuestionarios donde los alumnos expresen sus experiencias lectoras previas y su actitud hacia la literatura, así como sus concepciones iniciales sobre la lectura escolar y LIJ. Esta información contribuirá a que el profesorado universitario pueda elaborar planteamientos más realistas y más adaptados a las circunstancias propias de su alumnado. Díaz Armas (2008: 189) lo formula de manera muy clara al subrayar: "parece indispensable que conozcamos mejor las creencias del profesorado en proceso de formación inicial, y cómo estas creencias podrían operar sobre la práctica docente". En la información recogida, el profesorado de DLL ha destacado de forma significativa: el escaso hábito de lectura de los futuros docentes, algunos problemas de competencia lectora y literaria, cierta animadversión hacia la lectura y la literatura como consecuencia de experiencias didácticas previas poco gratificantes.
4. Dada la evidencia de la escasa formación lectora y literaria y de las experiencias previas con las que muchos de los futuros docentes llegan a la universidad, parece necesario introducir en la clases de DLL prácticas orientadas a incrementar su bagaje lector y propiciar situaciones que favorezcan un encuentro afectivo con la literatura y experiencias de lectura gratificantes, que necesariamente deberían ir acompañadas de procedimientos pertinentes de interpretación (Dueñas, Tabernero, Calvo y Consejo, 2014). Se trataría de potenciar en el aula experiencias de lectura estética (Rosenblatt, 2002), que tradicionalmente han estado descuidadas en la escuela y más aún en la universidad. En las respuestas del profesorado que participa en este trabajo, se destacan estas carencias casi de forma unánime y se subraya la dificultad que supone contar en el aula con estudiantes de Magisterio que no leen o a quienes no les guste leer. A pesar de los programas sobrecargados, del predominio de asignaturas cuatrimestrales que dejan poco tiempo al debate en el aula, resulta necesario que en las clases de Magisterio nuestros alumnos puedan leer, comentar, valorar, contrastar y debatir sobre las lecturas realizadas para que puedan encontrarse con la lectura literaria.

No tener en cuenta este factor sería muy negativo, ya que la labor de mediación no puede sustentarse en la mera búsqueda de procedimientos idóneos o de técnicas exitosas, sin considerar los aspectos personales (Mata, 2004) y, como bien afirma Chambers (2007), resulta muy difícil transmitir experiencias no vividas. En esta línea, Munita (2014) aboga por proponer itinerarios de lectura guiada, en los que el encuentro con la LIJ sería básico, para ampliar y diversificar los intertextos del futuro profesor. Yubero y Larrañaga (2015) plantean el desarrollo de programas de promoción lectora para estudiantes universitarios con propuestas orientadas a favorecer la lectura voluntaria, espacios para dialogar y compartir lecturas, y para favorecer la creación de comunidades de lectores (virtuales o no) desde la propia universidad y desde las bibliotecas universitarias. 
5. Asimismo, consideramos que los planteamientos didácticos de las materias de DLL deberían avanzar en el cambio de paradigma educativo que supone la introducción del enfoque competencial en la docencia de todos los niveles educativos. El aprendizaje, enfocado hacia la aplicabilidad de los conocimientos (Marco, 2008), debe traducirse en una manera específica de entender la educación y los procesos de enseñanza-aprendizaje (Cabrerizo, Rubio y Castillo, 2007). Supone "establecer el aprendizaje activo de conocimiento útil como el centro de la vida escolar" (Pérez Gómez, 2008: 86-87). El desarrollo de la competencia lectora y literaria en el marco de la competencia en comunicación lingüística y cultural no debe ser ajena a este planteamiento y debería abordarse en su complejidad, especialmente si se tiene en cuenta que la lectura recreativa regular resulta beneficiosa tanto para el desarrollo de la competencia lingüística como para el aprendizaje de otras materias (Merga, 2017). Como afirma Colomer (2014), todavía sigue siendo un tema pendiente el desarrollo de una programación por competencias que fusione los saberes literarios.

6. Una consecuencia de lo anterior es que las materias de DLL asuman un enfoque más práctico de la didáctica de la lectura y la literatura, donde se aborden aspectos más ligados a la realidad de las aulas de educación primaria, y a la dimensión personal y vivencial de la lectura y la literatura. Esto ayudaría a superar una visión excesivamente académica de éstas, concebida como lectura literaria-asignatura por gran parte del alumnado. Tal y como afirma Mendoza, frente a la disparidad de opciones metodológicas para abordar la educación literaria, "la actual orientación didáctica recoge cada vez con mayor precisión las razones para plantear la formación literaria en el espacio de la interacción y de la comunicación que se genera entre el texto literario y su lector" (Mendoza, 2005: 47-48).

Con cierta frecuencia los estudiantes de Magisterio perciben que una realidad es la DLL que se estudia en la universidad - entendida falsamente como algo exclusivamente teórico- - o otra bien distinta la que se hace en la escuela, de carácter eminentemente práctico. Por ello sería pertinente, tal y como plantea el profesorado encuestado, una mayor conexión entre la universidad y los centros de infantil, primaria y secundaria. También parece aconsejable reforzar líneas de actuación entre las materias cursadas en los centros universitarios y las prácticas docentes realizadas por el alumnado en los centros escolares durante el Practicum (Iñesta y Pascual, 2015), lejos de la realidad bastante habitual, según la cual el conocimiento práctico se supedita al teórico y al periodo de prácticas se le concede de hecho un papel formativo de segundo nivel (Mentado, Medina y Cruz, 2017). Por ello, resulta necesario que se mejore la coordinación entre los agentes responsables de la organización del Practicum y que se preste mayor atención a las competencias que debe desarrollar el alumnado (Cohen, Hoz y Kaplan, 2013). De forma especial, resultaría muy oportuno potenciar la realización de procesos de aprendizaje como reflexión-acción, en los cuales se establezcan redes de relación entre estudiantes y profesores en ejercicio respecto al tratamiento de la LIJ en la escuela (Díaz-Plaja y Prats, 2013).

7. Otra dimensión que necesariamente debe potenciarse más en la formación de los futuros docentes es la relacionada con los aspectos organizativos y de planificación que deben tomarse a nivel de centro educativo, asumiendo que la lectura, en todas sus facetas, es una cuestión de responsabilidad colectiva de toda la comunidad educativa. Los planes de lectura del centro (Pascual, 2012; Zayas y Lluch, 2015) y la biblioteca escolar (Durban, 2010) constituyen herramientas que pueden favorecer el desarrollo curricular y la innovación educativa con carácter general y, más en concreto, el desarrollo de la competencia lectora y literaria: aprender a leer, leer para aprender, gusto por la lectura y competencia informacional (Díaz-Plaja y Prats, 2013). Es preciso que en las clases de DLL se supere una visión de la educación lectora y literaria 
centrada casi exclusivamente en la mediación individual y se contemple esta dimensión institucional (Lerner, 2001). Esta consideración, si cabe, merece mayor atención ya que ha sido frecuentemente olvidada en las aulas universitarias. En relación con ello, los profesores de DLL de nuestro trabajo también demandan la necesidad de favorecer puntos de contacto entre las facultades y las organizaciones o instancias que promueven la lectura en su entorno: bibliotecas, librerías, autores de LIJ, etcétera.

\section{Referencias}

Aguilar, Consol (2017), "The DLL at the public University: an approach to the state of the question in the initial training of teachers. Part II", Lenguaje y textos, núm. 45, pp. 29-41.

Aguilar, Consol (2016), "La DLL en la universidad pública: una aproximación al estado de la cuestión en la formación inicial de maestros y maestros. Parte I", Lenguajeytextos, núm.44,pp.39-59, <http://repositori.uji. es/xmlui/bitstream/handle/10234/166700/14631. pdf? sequence $=6 \&$ isAllowed $=\mathrm{y}>$ [Consulta: enero de 2018].

Applegate, Anthony, Mary Dekonty Applegate, Marta A. Mercantini, Catherine M. McGeehan, Jeanne B. Cobb, Joanne R. DeBoy, Virginia Modla y Kimberly E. Lewinsky (2014), "The Peter effect revisited: reading habits and attitudes of college students", Literary research and instruction, vol. 53, núm. 3, pp. 188-204.

Arbonés, Carme, Antonio Mendoza, Soledad Muñoz, Margarida Prats y Eduard Sanahuja (2015), "Textualidades multimodales, conectividad y redes 2.0 en el aula: sobre la educación literaria y el profesorado en formación", en José Manuel de Amo, Osvaldo Cleger y Antonio Mendoza (eds.), Redes hipertextuales en
Este estudio ofrece resultados y conclusiones relevantes para el avance del campo, pero también presenta limitaciones propias de su enfoque: en futuros trabajos se hace necesario contrastar la perspectiva del profesorado con la perspectiva de los estudiantes y de los docentes en ejercicio de los centros escolares. En todo caso, pensamos que esta contribución puede ser muy relevante para el profesorado universitario que trabaja en el área de la DLL, al ofrecerle planteamientos y líneas de actuación que pueden redundar en la mejora de su función docente.

el aula. Literatura, hipertextos y cultura digital, Barcelona, Octaedro, pp. 97-120.

Ballester, Josep y Noelia Ibarra (2009), "La enseñanza de la literatura y el pluralismo metodológico", Ocnos, núm. 5, pp. 25-36, <https://doi.org/10.18239/ ocnos_2009.05.02>.

Ballester, Josep (2015), La formación lectora y literaria, Barcelona, Graó.

Cabrerizo, Jesús, María Julia Rubio y Santiago Castillo (2007), Programación por competencias: formación y práctica, Madrid, Prentice Hall.

Caldera, Reina, Dilia Escalante y Mirian Terán (2010), "Práctica pedagógica de la lectura y formación docente", Revista de Pedagogía, vol. 31, núm. 88, pp. 15-37, <http://www.redalyc.org/articulo. oa?id=65916617002> [Consulta: enero de 2018].

Camps, Anna (2012), "La investigación en didáctica de la lengua en la encrucijada de muchos caminos", Revista Iberoamericana de Educación, núm. 59, pp. 23-41, <https:// dialnet.unirioja.es/servlet/articulo?codigo $=4771116>$ [Consulta: enero de 2018].

Cerrillo, Pedro C. (2007), Literatura infantil y juvenil y educación literaria, Barcelona, Octaedro. 
Chambers, Aidan (2007), Dime. Los niños, la lectura y la conversación, México, Fondo de Cultura Económica.

Cohen, Esther, Ron Hoz y Haya Kaplan (2013), "The Practicum in preservice teacher education: a review of empirical studies", Teaching Education, vol. 24, núm. 4, pp. 345-380.

Colomer, Teresa (2014), "El aprendizaje de la competencia literaria”, en Carlos Lomas (ed.), La educación lingüística, entre el deseo y la realidad, Barcelona, Octaedro, pp. 110-122.

Colomer, Teresa (2001), "La enseñanza de la literatura como construcción de sentido", Lectura y vida. Revista Latinoamericana de lectura, vol. 22, núm. 4, pp. 2-19, <http://interactiva.eafit.edu.co/ei/contenido/ micrositios/1918943/Lengua_escrita_Aula2/ documentos/Colomer_Ensenanza_literatura.pdf $>$ [Consulta: enero de 2018].

Colomer, Teresa y Felipe Munita (2013), "La experiencia lectora de los alumnos de Magisterio: nuevos desafios para la formación docente", Lenguaje y Textos, núm. 38, pp. 37-44, <http://www.sedll.org/sites/default/files/ journal/la_experiencia_lectora_de_los_alumnos_de_ magisterio._colomer_t.pdf> [Consulta: enero de 2018].

Cremin, Teresa, Marilyn Mottram, Eve Bearne y Prue Goodwin (2008), "Exploring teachers knowledge of children's literature", Cambridge Fournal of Education, vol. 38, núm. 4, pp. 449-464.

Díaz Armas, Jesús (2008), "Lectura literaria y formación inicial. Creencias del profesorado en formación sobre el texto y su lector", en Antonio Mendoza (coord.), Textos entre textos: las conexiones textuales en la formación del lector, Barcelona, Horsori, pp. 177-190.

Díaz, Juan P., Aníbal R. Bar y Margarita C. Ortiz (2015), "La lectura crítica y su relación con la formación disciplinar de estudiantes universitarios", Revista de la Educación Superior, vol. XLIV (4); núm. 176, pp. 139-158, <http://www.scielo.org.mx/pdf/resu/ v44n176/v44n176a7.pdf> [Consulta: enero de 2018].

Díaz-Plaja, Ana y Margarida Prats (2013), "Recordar, aprender, practicar: qué sabe y qué debe saber un futuro maestro", Lenguaje y Textos, núm. 38, pp. 19-28, <http://www.sedll.org/sites/default/files/journal/ recordar_aprender_practicar_que_sabe_y_que_ debe_saber_un_futuro_maestro._diaz-plaja_a._y_ ot.pdf> [Consulta: enero de 2018].

Dueñas, José D., Rosa Tabernero, Virginia Calvo y Elena Consejo (2014), "La lectura literaria ante nuevos retos: canon y mediación en la trayectoria lectora de futuros profesores", Ocnos, núm. 11, pp. 21-43, $<$ https://revista.uclm.es/index.php/ocnos/article/ view/ocnos_2014.11.02/434> [Consulta: enero de 2018].

Durban, Gloria (2010), La biblioteca escolar hoy: un recurso estratégico para el centro, Barcelona, Graó.

Felipe, Andrea (2016), "Competencia, estrategias y hábitos lectores de maestros en formación inicial" (tesis doctoral), Málaga, Universidad de Málaga, <https:// riuma.uma.es/xmlui/handle/10630/14184> [Consulta: enero de 2018].

Granado, Cristina y María Puig (2014), “¿Qué leen los futuros maestros y maestras? Un estudio del docente como sujeto lector a través de los títulos de libros que evocan", Ocnos, núm. 11, pp. 93-112, <https://www. revista.uclm.es/index.php/ocnos/article/viewFile/ ocnos_2014.11.05/427> [Consulta: enero de 2018].

Granado, Cristina (2014), "Teachers as readers: a study of the reading habits of future teachers/El docente como lector: estudio de los hábitos lectores de futuros maestros", Cultura y Educación/Culture and Education, vol. 26, núm. 1, pp. 44-70.

Gutiérrez-Fresneda, Raúl, Antonio Díez-Mediavilla y Elena Jiménez-Pérez (2017), "Estudio longitudinal sobre el aprendizaje lector en las primeras edades", Revista de Educación, núm. 378, pp. 30-51, <https:// www.mecd.gob.es/dctm/revista-de-educacion / articulos378/02gutierrez.pdf?documentId=0901e72b 82657abb> [Consulta: enero de 2018].

Hoeft, Mary E. (2012), "Why university students don't read: what professors can do to increase compliance", International Fournal for the Scholarship of Teaching and Learning, vol. 6, núm. 2, pp. 1-20, <https://files.eric. ed.gov/fulltext/EJ1135576.pdf > [Consulta: enero de 2018]. 
Iñesta, Eva M. y Julián Pascual (2015), "Didáctica para el plurilingüismo en la formación de maestros: estudio empírico desde el Prácticum”, Aula Abierta, núm. 43, pp. 94-101, <http://www.elsevier.es/es-revista-aulaabierta-389-articulo-didactica-el-plurilinguismoformacion-maestros-S $0210277314000390>$ [Consulta: enero de 2018].

Larrañaga, Elisa, Santiago Yuberoy Pedro Cerrillo(2008), Estudio sobre los hábitos de lectura de los universitarios españoles, Madrid, SM.

Leland, Katina M. (2013), "The impact of a teacher preparation literacy course on pre-service teachers' perceptions of teaching young children how to read", SRATE Fournal, vol. 22, núm. 2, pp. 65-70, <https:// files.eric.ed.gov/fulltext/EJ1015816.pdf> [Consulta: enero de 2018].

Lerner, Delia (2001), Leer y escribir en la escuela: lo real, lo posible y lo necesario, México, Fondo de Cultura Económica.

Marco, Berta (2008), Competencias básicas. Hacia un nuevo paradigma educativo, Madrid, Narcea/MEPSyD.

Mata, Juan (2008), 10 ideas clave. Animación a la lectura, Barcelona, Graó.

Mata, Juan (2004), Cómo mirar a la luna (confesiones a una maestra sobre la formación del lector, Barcelona, Graó.

Mendoza, Antonio (2011), "La investigación en didáctica de las primeras lenguas", Educatio Siglo XXI, vol. 29, núm. 1, pp. 31-80, <http://revistas.um.es/educatio/ article/view/119871> [Consulta: enero de 2018].

Mendoza, Antonio (2005), "La educación literaria desde la literatura infantil y juvenil", en Carmen Utanda, Pedro Cerrillo y Jaime García Padrino (eds.), Literatura infantil y educación literaria, Cuenca, Universidad de Castilla-La Mancha, pp. 33-61.

Mentado, Trinidad, José Luis Medina y Lorena Cruz (2017), "Preparar para aprender: una manifestación del conocimiento didáctico del contenido en la práctica", Estudios sobre Educación, vol. 33, pp. 27-48, <https:// www.unav.edu/publicaciones/revistas/index.php/ estudios-sobre-educacion/article/view/11565/9038> [Consulta: enero de 2018].
Merga, Margaret K. (2017), "What would make children read for pleasure more frequently?", English in Education, vol. 51, núm. 2, pp. 207-223.

Munita, Felipe (2014), "Reading habits of pre-service teachers/Trayectorias de lectura del profesorado en formación", Cultura y Educación, vol. 26, núm. 3, pp. 448-475, <DOI:10.1080/11356405.2014.96544>.

Munita, Felipe (2013), "Creencias y saberes de futuros maestros (lectores y no lectores) en torno a la educación literaria", Ocnos, núm. 9, pp. 69-87, <https:// www.revista.uclm.es/index.php/ocnos/article/ viewFile/227/257> [Consulta: enero de 2018].

Pascual,Julián (2012), "Plan Lector de Centro y biblioteca escolar: dos herramientas para la innovación educativa", Textos de Didáctica de la Lengua y la Literatura, núm. 60, pp. 13-22.

Pérez Gómez, Ángel I. (2008), “CCompetencias o pensamiento práctico? La construcción de los significados de representación y de acción", en José Gimeno (ed.), Educar por competencias, ¿qué hay de nuevo?, Madrid, Morata, pp. 59-102.

Rosenblatt, Louise M. (2002), La literatura como exploración, México, Fondo de Cultura Económica.

Serafini, Frank (2012), "Reading multimodal texts in the 21th Century", Research in the Schools, vol. 19, núm. 1, pp. 26-32, <https://pdfs.semanticscholar. org/5c28/a5f4391bc083f03ba186bld573fle3c131d1. pdf $>$ [Consulta: enero de 2018].

Tabernero, Rosa (2013), "El lector literario en los grados de Maestro: deconstruir para construir", Lenguaje y Textos, núm. 38, pp. 47-56, <http://www.sedll. org/sites/default/files/journal/el_lector_literario_ en_los_grados_de_maestro_deconstruir_para_ construir._tabernero_r.pdf $>$ [Consulta: enero de 2018].

Vidal-Moscoso, Daniela y Leonardo Manríquez-López (2016), "El docente como mediador de la comprensión lectora en universitarios", Revista de la Educación Superior, vol. XLV (1), núm. 177, pp. 95-118, <http:// publicaciones.anuies.mx/pdfs/revista/Revista177_ S3A4ES.pdf> [Consulta: enero de 2018]. 
Yubero, Santiago y Elisa Larrañaga (2015), "Lectura y universidad: hábitos lectores de los estudiantes universitarios de España y Portugal", El profesional de la información, vol. 24, núm. 6, pp. 717-723, <http://www. elprofesionaldelainformacion.com/contenidos/2015/ nov/03.pdf> [Consulta: enero de 2018].
Zayas, Felipe y Gemma Lluch (2015), "Ideas clave para orientar los planes de lectura y escritura", Textos de Didáctica de la Lengua y la Literatura, núm. 68, pp. 9-17.

\section{Cómo citar este artículo:}

Álvarez-Álvarez, Carmen y Julián Pascual-Díez (2020), "Formación inicial de maestros en promoción de la lectura y la literatura en España desde la perspectiva del profesorado universitario", Revista Iberoamericana de Educación Superior (RIES), vol. XI, núm. 30, pp. 57-75, https://doi.org/10.22201/iisue.20072872e.2020.30.588 [Consulta: fecha de última consulta]. 